Review article based on an invited lecture given at the NATO Advanced Study Institute

“The Neutron Star - Black Hole Connection” June 7-18 1999, Elounda, Crete.

\title{
NEUTRON STAR BIRTH RATES
}

\author{
D.R. LORIMER \\ National Astronomy and Ionospheric Center \\ Arecibo Observatory \\ HC3 Box 53995, Arecibo PR 00612, USA
}

\begin{abstract}
A crucial test any proposed evolutionary scenario must pass is can the birth rate of the sources we see be sustained by the proposed progenitor population? In this review, I investigate the methods used to determine the birth rates of normal and millisecond radio pulsars and summarise recent results for these two distinct neutron star populations.
\end{abstract}

\section{Introduction}

In principle, estimating the birth rate of a population of sources is trivial: divide the total number of sources by the mean lifetime of the population. In practice, however, for the neutron star population, precise estimates of both the number and lifetime of the sources are hard to obtain. The sample of Galactic neutron stars is heavily biased by a number of observational selection effects which must be properly accounted for in a birth rate analysis.

In this review I shall be mainly concerned with the birth rates of two distinct sub-populations of the neutron star zoo: the normal pulsars and the millisecond pulsars. $\S 2$ defines what is meant by these two classes. $\S 3$ reviews the "standard" evolutionary scenarios which connect normal pulsars to supernova remnants and millisecond pulsars to low-mass X-ray binaries. $\S 4$ discusses the various selection effects known to significantly bias these populations. $§ 5$ summarises techniques to correct for these effects. This leads naturally to an estimate of the birth rates (\$6) which are discussed in context with the proposed progenitor populations in $\S 7$. Some suggestions for future work are also made in this section. 


\section{Normal and Millisecond Pulsars}

As I alluded to in my other contribution to this volume, the period $P$ and period derivative $\dot{P}$ for each pulsar can be obtained to very high levels of precision through a series of timing measurements. Perhaps the most oft-plotted figure from these data is the " $P-\dot{P}$ diagram" shown in Fig. 1.

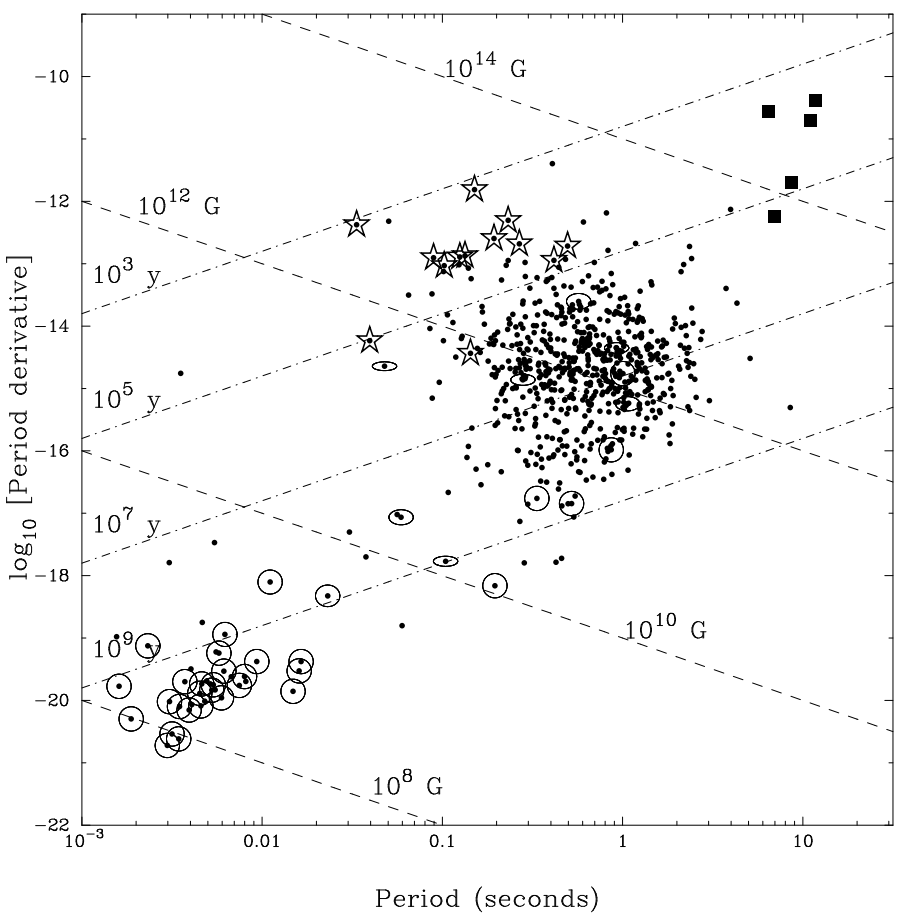

Figure 1. The ubiquitous $P-\dot{P}$ diagram shown for a sample consisting of radio pulsars (the black dots) and anomalous X-ray pulsars (the black squares - see review by Mereghetti in this volume). Pulsars known to be members of binary systems are highlighted by a circle (for low-eccentricity orbits) or an ellipse (for elliptical orbits). Pulsars thought to be associated with supernova remnants are highlighted by the starred symbols.

Amongst other things, the diagram demonstrates clearly the distinction between the "normal pulsars" $\left(P \sim 0.5 \mathrm{~s}\right.$ and $\dot{P} \sim 10^{-15} \mathrm{~s} / \mathrm{s}$ and populating the "island" of points) and the "millisecond pulsars" $(P \sim 3 \mathrm{~ms}$ and $\dot{P} \sim$ $10^{-20} \mathrm{~s} / \mathrm{s}$ and occupying the lower left part of the diagram).

The differences in $P$ and $\dot{P}$ imply fundamentally different typical ages and magnetic field strengths for the two populations. For the "canonical neutron star" of mass $1.4 \mathrm{M}_{\odot}$, radius $10 \mathrm{~km}$ and moment of inertia $10^{45} \mathrm{~g} \mathrm{~cm}^{2}$ (see for example Shapiro \& Teukolsky 1983), it can be shown that the surface dipole magnetic field strength in units of $10^{12}$ Gauss, $B_{12} \simeq\left(P_{s} \dot{P}_{-15}\right)^{1 / 2}$, where $P_{s}$ is the period in seconds and $\dot{P}_{-15}=10^{15} \dot{P}$. Integrating this first-order differential equation over time, assuming a con- 
stant magnetic field, results in an expression for the age of the pulsar $t=\tau\left[1-\left(P_{0} / P\right)^{2}\right]$, where $\tau=P / 2 \dot{P}$ is the so-called "characteristic age" and $P_{0}$ is the period of the pulsar at $t=0$. Under the assumption that the neutron star has slowed down significantly since birth $\left(P_{0} \ll P\right)$, the characteristic age $\tau$ is a good approximation to the true age $t$. Lines of constant $B$ and $\tau$ are drawn on Fig. 1. From these, we infer typical magnetic fields and ages of $10^{12} \mathrm{G}$ and $10^{7} \mathrm{yr}$ for the normal pulsars and $10^{8} \mathrm{G}$ and $10^{9} \mathrm{yr}$ for the millisecond pulsars respectively.

Whilst the consensus of evidence generally supports the assumptions in the characteristic age estimates, viz: dipolar spin down from a small initial spin period and a constant magnetic field (see e.g. Bhattacharya et al. 1992; Lorimer et al. 1993), we should caution that, rather like dispersion-measurederived distances, individual characteristic ages should not be taken as precise values. It is therefore of interest to look for independent age constraints. Strong support of the characteristic age estimates for old pulsars comes from the observations of asymmetric drift seen in the proper motions of normal pulsars with large characteristic ages (Hansen \& Phinney 1997), and overwhelmingly in the millisecond pulsars (Toscano et al. 1999). See Ramachandran's review in this volume for further discussion.

A very important additional difference between normal and millisecond pulsars is binarity. Orbital companions are much more commonly observed around millisecond pulsars ( $\sim 80 \%$ of the observed sample) than the normal pulsars $(\lesssim 1 \%)$. As discussed in my other contribution to these proceedings, timing measurements constrain the masses of orbiting companions which, when supplemented by observations at other wavelengths, tell us a great deal about their nature. The present sample of orbiting companions are either white dwarfs, main sequence stars, or other neutron stars. Binary pulsars with low-mass companions $\left(\lesssim 0.5 \mathrm{M}_{\odot}\right.$ - predominantly white dwarfs) usually have millisecond spin periods and essentially circular orbits: $10^{-5} \lesssim e \lesssim 10^{-1}$. Measurements of the "cooling ages" of the white dwarfs (see e.g. van Kerkwijk 1996) provide further evidence that millisecond pulsars have typical ages of a few Gyr. The binary pulsars with high-mass companions ( $\gtrsim 1 \mathrm{M}_{\odot}$ - neutron stars or main sequence stars) have larger spin periods ( $\gtrsim 30 \mathrm{~ms}$ ) and are in much more eccentric orbits: $0.2 \lesssim e \lesssim 0.9$.

\section{Evolutionary Scenarios}

We now briefly review the various end-products that are implied by standard models for the formation and evolution of single and binary radio pulsars which basically assume that every neutron star in the disk of our Galaxy was formed during the core-collapse phase of a supernova explosion of a massive star (see also van den Heuvel's contribution to this volume). 
The simplest scenario is shown in Fig. 2 and begins in the final moments of the life of a single massive star that is about to explode as a supernova. The neutron star formed during the core collapse will receive an impulsive

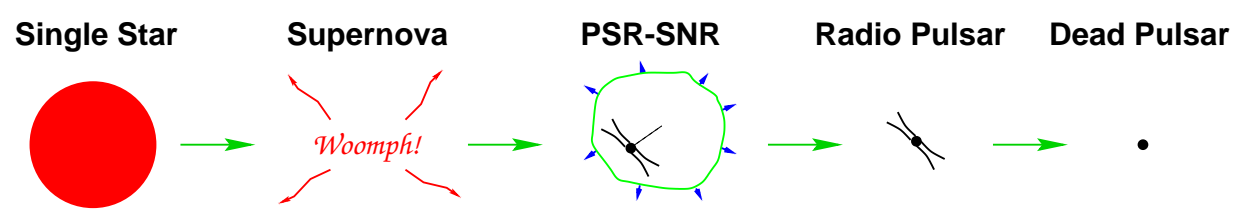

Figure 2. Cartoon showing the evolutionary sequence of a single neutron star.

kick (see e.g. Spruit \& Phinney 1998) if the explosion is not symmetric and, as a result, begin to move away from the centre of the explosion. In the meantime, the outer layers of the star are expanding into the surrounding space at velocities of up to $10,000 \mathrm{~km} \mathrm{~s}^{-1}$. The result is a pulsar-supernova remnant association (PSR-SNR) which may be visible as a pair for up to $10^{5} \mathrm{yr}$ after the explosion. Eventually, the expanding shell becomes so diffuse that it is no longer visible as a supernova remnant. The pulsar, on the other hand, may produce radio emission for a further $10^{7} \mathrm{yr}$ or more as it gradually spins down to longer periods. Presently, the longest period for a radio pulsar is $8.5 \mathrm{~s}$ (Young, Manchester \& Johnston 1999). At some point the rotational energy of the neutron star is insufficient to induce pair production in its magnetosphere and the radio emission ceases.

The presently favoured model to explain the formation of the various types of binary systems has been developed over the years by a number of authors (Bisnovatyi-Kogan \& Komberg 1974; Flannery \& van den Heuvel 1975; Smarr \& Blandford 1976; Alpar et al. 1982). The model is sketched in Fig. 3 and can be qualitatively summarised as follows: starting with a binary star system, the neutron star is formed during the supernova explosion of the initially more massive star (the primary) which has an inherently shorter main sequence lifetime. From the virial theorem, it follows that the binary system gets disrupted if more than half the total pre-supernova mass is ejected from the system during the explosion. In addition, the fraction of surviving binaries is affected by the magnitude and direction of the impulsive kick velocity the neutron star receives at birth (Hills 1983; Bailes 1989). Those binary systems that disrupt produce a high-velocity isolated neutron star and an OB runaway star (Blaauw 1961). Rather like the fortunate survivor of a crash, the isolated pulsar has no recollection of the binary system it once belonged to and behaves from the moment of release as a single pulsar discussed above. The high binary disruption probability explains why so few normal pulsars are observed with orbiting companions. 


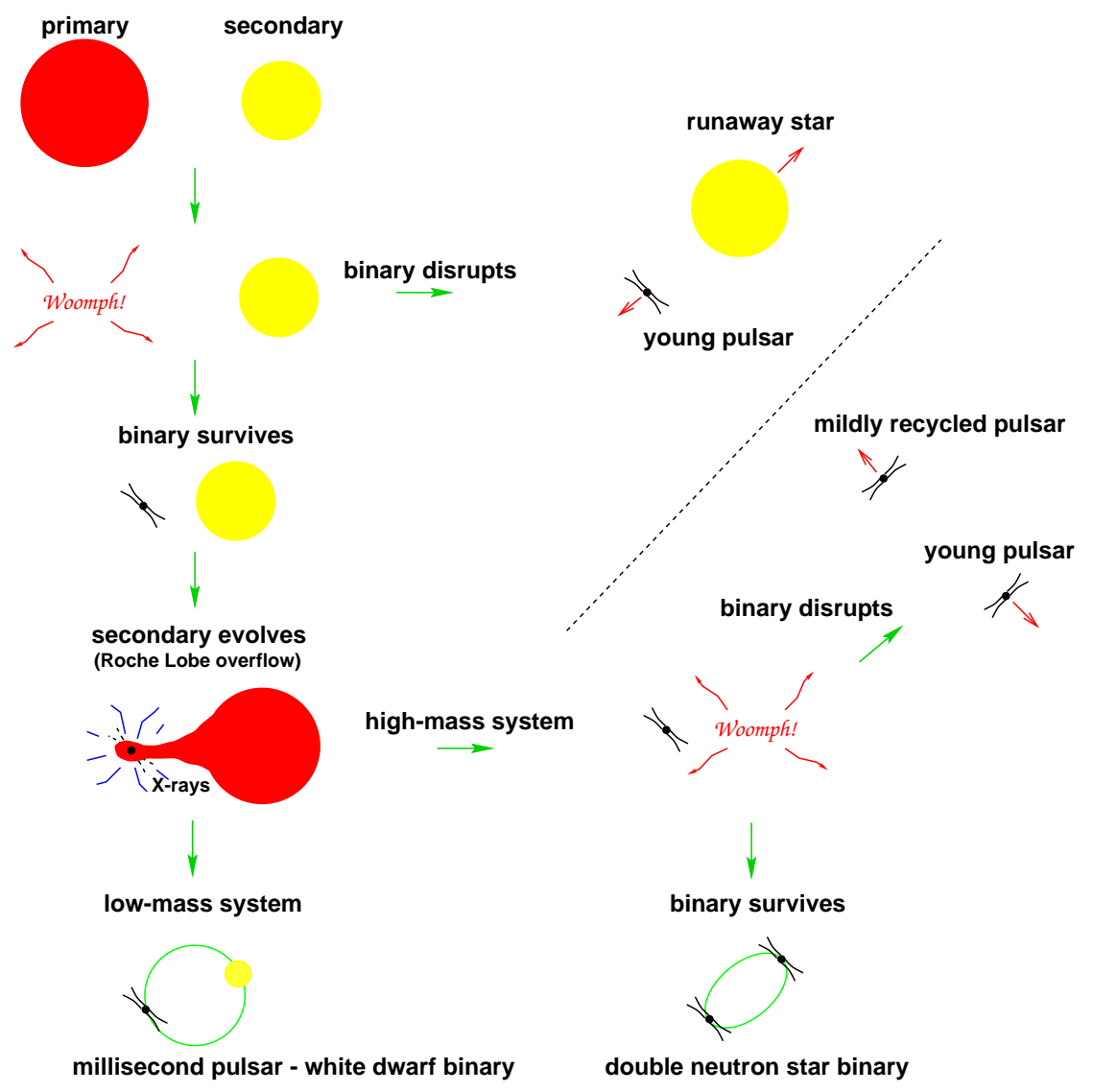

Figure 3. Cartoon showing various evolutionary scenarios involving binary pulsars.

For those few binaries that remain bound, and where the companion star is sufficiently massive to evolve into a giant and overflow its Roche lobe, the old spun-down neutron star can gain a new lease of life as a pulsar by accreting matter and therefore angular momentum from its companion (Alpar et al. 1982). The term "recycled pulsar" is often used to describe such objects. During this accretion phase, the X-rays liberated by heating the material falling onto the neutron star mean that such a system is expected to be visible as an X-ray binary system.

Two classes of X-ray binaries relevant to binary and millisecond pulsars exist: neutron stars with high-mass or low-mass companions. The highmass companions are massive enough to explode themselves as a supernova, producing a second neutron star. If the binary system is lucky enough to survive this explosion, it ends up as a double neutron star binary. The classic example is PSR B1913+16, a 59-ms radio pulsar with a characteristic age of $\sim 10^{8}$ yr which orbits its companion every $7.75 \mathrm{hr}$ (Hulse \& 
Taylor 1975). In this formation scenario, PSR B1913+16 is an example of the older, first-born, neutron star that has subsequently accreted matter from its companion. Presently, there are no clear observable examples of the second-born neutron star in these systems. This is probably reasonable when one realises that the observable lifetimes of recycled pulsars are much larger than normal pulsars. As discussed by Kalogera in this volume, double neutron star binary systems are very rare in the Galaxy - another indication that the majority of binary systems get disrupted when one of the components explodes as a supernova. Systems disrupted after the supernova of the secondary form a midly-recycled isolated pulsar and a young pulsar (formed during the explosion of the secondary).

By definition, the companions in the low-mass X-ray binaries evolve and transfer matter onto the neutron star on a much longer time-scale, spinning the star up to periods as short as a few ms (Alpar et al. 1982). This model has recently gained strong support from the detection of Doppler-shifted 2.49-ms X-ray pulsations from the transient X-ray burster SAX J1808.43658 (Wijnands \& van der Klis 1998; Chakrabarty \& Morgan 1998). At the end of the spin-up phase, the secondary sheds its outer layers to become a white dwarf in orbit around a rapidly spinning millisecond pulsar. A number of binary millisecond pulsars now have compelling optical identifications of the white dwarf companion. The existence of solitary millisecond pulsars in the Galactic disk (which comprise just under $20 \%$ of all Galactic millisecond pulsars) cannot easily be explained in the context of this model and alternative formation scenarios need to be developed.

\section{Selection Effects}

Having gotten a flavour for the likely evolutionary scenarios, we now turn our attention to the first piece in the birth rate puzzle - how many active radio pulsars exist in the Galaxy? Although over 1200 sources are presently known, the total Galactic population is hidden from us by a number of selection effects. As a result, the true number of pulsars is likely to be substantially larger than the observed number.

The most prominent selection effect at play in the observed pulsar sample is the inverse square law, i.e. for a given luminosity the observed flux density falls off as the inverse square of the distance. This results in the observed sample being dominated by nearby and/or bright objects. This effect is well demonstrated by the clustering of known pulsars around our location when projected onto the Galactic plane shown in Fig. 4. Although this would be consistent with Ptolemy's geocentric picture of the heavens, it is clearly at variance with what we now know about the Galaxy, where the massive stars show a radial distribution about the Galactic centre. 
The extent to which the sample is incomplete is shown in the right panel of Fig. 4 where the cumulative number of pulsars is plotted as a function of the projected distance from the Sun. The observed distribution (solid line) is compared to the expected distribution (dashed line) for a uniform disk population in which there are errors in the distance scale, but no such selection effects. We see that the observed sample becomes strongly deficient in terms of the number of sources for distances beyond a few kpc.

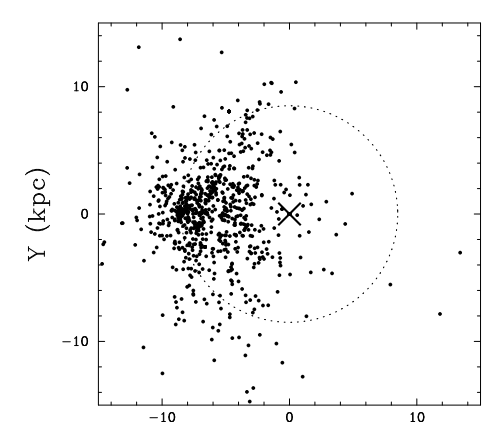

$\mathrm{X}(\mathrm{kpc})$

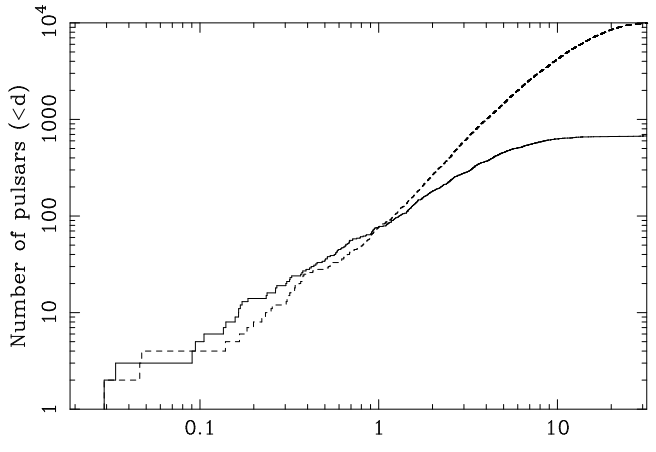

Projected Distance from the Sun $(\mathrm{kpc})$

Figure 4. Left: the observed sample of pulsars projected onto the Galactic plane. The Galactic centre is at $(0,0)$ and the Sun is at $(-8.5,0)$. Right: Cumulative number of observed pulsars (solid line) as a function of projected distance from the Sun. The dashed line shows the expected distribution for a hypothetical uniform disk Galaxy (see text).

Beyond distances of a few kpc from the Sun, the apparent flux density falls below the flux thresholds $S_{\min }$ of most surveys. Following Dewey et al. (1984), we may parameterise the survey threshold by:

$$
S_{\min }=\frac{\beta \sigma_{\min }\left(T_{\mathrm{rec}}+T_{\mathrm{sky}}\right)}{A_{e} \sqrt{N_{p} \Delta \nu t_{\mathrm{int}}}} \sqrt{\frac{W}{P-W}} .
$$

In this expression $\beta$ is a correction factor $\sim 1.3$ which reflects losses to hardware limitations, $\sigma_{\min }$ is the threshold signal-to-noise ratio (typically $7-10), T_{\text {rec }}$ and $T_{\text {sky }}$ are the receiver and sky noise temperatures $(\mathrm{K}), A_{e}$ is the effective area of the antenna in K/Jy $\left(1 \mathrm{~K} / \mathrm{Jy}=2760 \mathrm{~m}^{2}\right), N_{p}$ is the number of polarisations observed, $\Delta \nu$ is the observing bandwidth, $t_{\text {int }}$ is the integration time, $W$ is the detected pulse width and $P$ is the pulse period. This expression is valid as long as $W<P$.

If the detected pulse width $W$ equals the pulse period, the pulsar is of course no longer detectable as a periodic radio source. The detected pulse width is larger than the intrinsic value for a number of reasons: finite sampling effects, pulse dispersion, as well as scattering due to the presence of free electrons in the interstellar medium. From Eq. 1 of my other article 
in this volume, it is easy to show that the dispersive smearing scales as $\Delta \nu / \nu^{3}$, where the bandwidth $\Delta \nu$ is assumed to be much smaller than $\nu$, the observing frequency. As discussed in the other article, this can largely be removed via the de-dispersion process. The smearing across the individual channels, however, still remains and becomes significant when searching for short-period $(P \lesssim 200 \mathrm{~ms})$ pulsars located at large distances.

As well as the dispersion broadening effect, free electrons in the interstellar medium can scatter the pulses causing an additional broadening due to the different arrival times of scattered pulses. A simple scattering model is shown in Fig. 5 in which the scattering electrons are assumed to lie in a thin screen between the pulsar and the observer (Scheuer 1968). At ob-

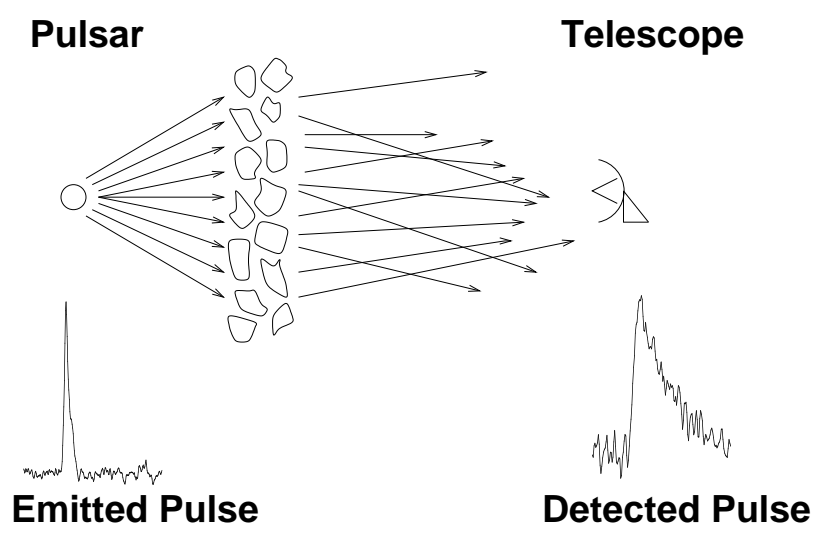

Figure 5. Pulse scattering caused by irregularities in the interstellar medium. The difference in path lengths and therefore in arrival times of the scattered rays result in a "scattering tail" in the observed pulse profile which lowers its signal-to-noise ratio.

serving frequencies $\lesssim 400 \mathrm{MHz}$, scattering becomes particularly important for pulsars with DMs $\gtrsim 200 \mathrm{~cm}^{-3} \mathrm{pc}$. The increased column density of free electrons can cause a significant tail in the observed pulse profile as shown in Fig. 5, reducing the effective signal-to-noise ratio and overall search sensitivity. Multi-path scattering results in a one-sided broadening due to the delay in arrival times which scales roughly as $\nu^{-4}$. This cannot be removed by instrumental means.

Dispersion and scattering become most severe for distant pulsars in the inner Galaxy as the number of free electrons along the line of sight becomes large. The strong inverse frequency dependence of both effects means that they are considerably less of a problem for surveys at observing frequencies $\gtrsim 1400 \mathrm{MHz}$ compared to the usual $400 \mathrm{MHz}$ search frequency. An added bonus for such observations is the reduction in $T_{\text {sky }}$, since the spectral index of the non-thermal emission is about -2.8 (Lawson et al. 1987). Pulsars themselves have steep radio spectra. Typical spectral indices are 
-1.6 (Lorimer et al. 1995), so that flux densities are an order of magnitude lower at $1400 \mathrm{MHz}$ compared to $400 \mathrm{MHz}$. This can usually be compensated somewhat by the use of larger receiver bandwidths at higher radio frequencies. The tremendous success of the multibeam surveys at Parkes is due to a combination of all these factors which allow the survey to probe deeper into the Galaxy than has been possible hitherto (Camilo et al. 2000).

A selection effect that we simply have to live with is beaming: the fact that the emission beams of radio pulsars are narrow means that only a fraction of $4 \pi$ steradians is swept out by the radio beam during one rotation. A first-order estimate of the so-called "beaming factor" or "beaming fraction" $(f)$ is $20 \%$; this assumes a beam width of 10 degrees and a randomly distributed inclination angle between the spin and magnetic axes.

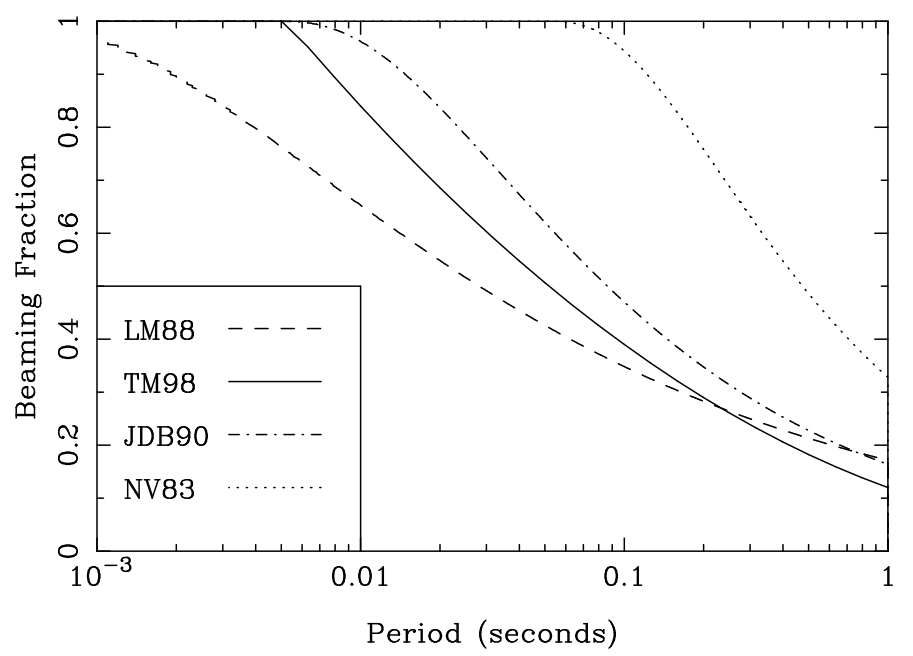

Figure 6. Beaming fraction plotted against pulse period for four different beaming models: Tauris \& Manchester (1998; TM88), Lyne \& Manchester (1988; LM88), Biggs (1990; JDB90) and Narayan \& Vivekanand (1983; NV83).

More detailed studies show that short-period pulsars have wider beams and therefore larger beaming fractions than their long-period counterparts (Narayan \& Vivekanand 1983; Lyne \& Manchester 1988; Biggs 1990; Tauris \& Manchester 1998). It must be said, however, that a consensus on the beaming fraction-period relation has yet to be reached. This is shown in Fig. 6 where we compare the period dependence of $f$ as given by a number of models. Adopting the Lyne \& Manchester model, pulsars with periods $\sim 0.1 \mathrm{~s}$ beam to about $30 \%$ of the sky compared to the Narayan \& Vivekanand model in which pulsars with periods below $0.1 \mathrm{~s}$ beam to the entire sky. When many of these models were proposed, the sample of millisecond pulsars was $\lesssim 5$ and hence their predictions about the beaming 
fractions of short-period pulsars relied largely on extrapolations from the normal pulsars. A recent analysis of a large sample of millisecond pulsar profiles (Kramer et al. 1998) suggests that the beaming fraction of millisecond pulsars lies between 50 and $100 \%$.

Before concluding this section, it is appropriate to mention two other selection effects which we will not discuss in detail: smearing of the pulse due to motion in a binary system, and nulling of long-period pulsars. The former effect is discussed in part in my other contribution to these proceedings and is most relevant to the double neutron star population (see Kalogera, this volume). The latter effect, nulling, refers to the apparent spasmodic switch off in the pulsar emission process first observed by Backer (1970) in a study of single pulses at Arecibo. The obvious connotation here is that pulsars which spend significant periods in the "null state" will be missed by any systematic searches, particularly those with short integration times. For example, the existence of PSR B0826-34, was extremely difficult to confirm since its null state often lasts many hours (Durdin et al. 1979).

In the context of neutron star birth rates, the selection effect caused by pulse nulling is of little significance, however, since it is fairly certain from the work of e.g. Ritchings (1976) that nulling occurs predominantly in older, long-period pulsars. In the context of pulsar surveys in general, however, this effect is potentially important. Nice (1999) has recently reanalysed archival Arecibo search data to look for dispersed single pulses. By comparison to the Fourier-transform-based searches outlined in my other article, the search analysis employed by Nice was trivial. One new 2-s pulsar, J1918+08, that emitted only one detectable pulse (!) during the original survey integration (67 s) was discovered. Nice's results suggest that further long-period pulsars which only occasionally emit detectable pulses may be found by future searches with only a modest amount of additional effort.

\section{Accounting for Selection Effects}

Now that we know how severe the selection effects are, how do we correct for them? There are three basic techniques: (1) source counting; (2) Monte Carlo simulations; (3) scale factor calculations.

Counting sources (see e.g. Kundt 1992) is by far the crudest method, but nevertheless easy and instructive. In essence the trick is to look at the cumulative distribution shown in Fig. 4 and count sources out to a distance where you think the sample is reasonably complete and then, by assuming some underlying distribution function, extrapolate this number to get the total number of sources in the Galaxy. Based on Fig. 4 we count 100 objects out to $1 \mathrm{kpc}$, i.e. a mean surface density of $100 /\left(\pi \times 1 \mathrm{kpc}^{2}\right) \simeq 30 \mathrm{kpc}^{-2}$. If pulsars have a radial distribution similar to that of other stellar popu- 
lations, the corresponding local-to-Galactic scale factor is then $1000 \pm 250$ $\mathrm{kpc}^{2}$ (Ratnatunga \& van den Bergh 1989). With this factor, we estimate there to be of order 30,000 potentially observable pulsars in the Galaxy. Assuming a beaming fraction of $20 \%$ scales this to a total of 150,000 .

Whilst this simple technique gives a rough answer, and can be done on the back of an envelope, it is clearly not making good use of all the available information contained in the observed sample. A considerably more rigorous approach, shown in Fig. 7, is to carry out a Monte Carlo

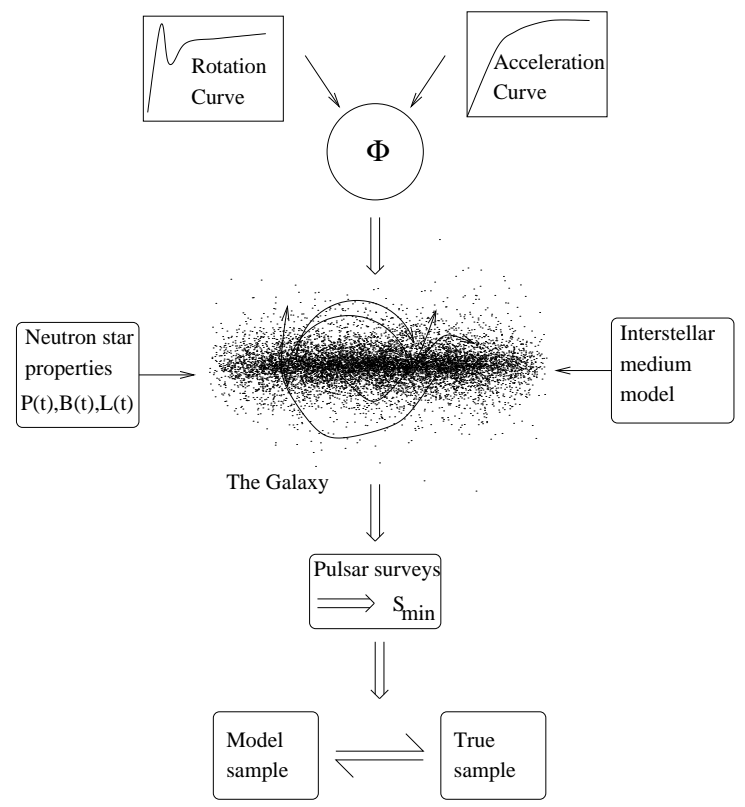

Figure 7. The Full Monty - an idealised neutron star population synthesis scheme.

simulation of the Galaxy, the insterstellar medium and the neutron star population. Numerous accounts of this technique can be found in the literature, see e.g. Stollman (1987); Narayan (1987); Emmering \& Chevalier (1989); Narayan \& Ostriker (1990); Bhattacharya et al. (1992).

In brief, the simulation seeds a model galaxy with pulsars having a given set of initial parameters (period, magnetic field strength, space velocity). The model pulsars are then allowed to evolve kinematically in a model of the Galactic gravitational potential, and rotationally (based on a model for pulsar spin-down and luminosity) for a given time. The detailed models of the pulsar surveys then produce a "model observed sample" which can then be directly compared to the sample of 1200 objects that we actually observe. By varying the input model parameters and repeating the simulation to maximise the agreement between the model and actually observed samples, 
it is possible to directly constrain the physical parameters of the Galactic neutron star population.

Although these Monte Carlo simulations assume a pulsar birth rate, and in this sense can be used to obtain a "best-fitting value", problems are often encountered in dealing with the large number of assumptions required about pulsar spin-down and the radio luminosity evolution. What these simulations do best is to teach us about selection effects and allow useful tests of ideas hypothesised from the observable population e.g. magnetic field decay (Bhattacharya et al. 1992) and the velocity-magnetic moment correlation (Lorimer, Bailes \& Harrison 1997).

The most model-independent way of constraining the number of pulsars in the Galaxy and ultimately the birth rate is, following Phinney \& Blandford (1981) and Vivekanand \& Narayan (1981), to define a scaling function $\xi$ as the ratio of the total Galactic volume weighted by pulsar density to the volume in which a pulsar could be detected by various systematic surveys:

$$
\xi(P, L)=\frac{\iint_{\text {Galaxy }} \Sigma(R, z) R d R d z}{\iint_{P, L} \Sigma(R, z) R d R d z} .
$$

In this expression, $\Sigma(R, z)$ is the assumed pulsar density in terms of galactocentric radius $R$ and height above the Galactic plane $z$. Note that $\xi$ is primarily a function of period $P$ and luminosity $L$ such that short period/lowluminosity pulsars have smaller detectable volumes and therefore higher $\xi$ values than their long-period/high-luminosity counterparts.

The scaling function is calculated in practice for each pulsar individually using a Monte Carlo simulation to model the volume of the Galaxy probed by the major surveys (Narayan 1987). For a sample of $N_{\text {obs }}$ observed pulsars above a minimum luminosity $L_{\min }$, the total number of pulsars in the Galaxy with luminosities above this value is

$$
N_{\text {gal }} \approx \sum_{i=1}^{N_{\text {obs }}} \frac{\xi_{i}}{f_{i}}
$$

where $f$ is the model-dependent beaming fraction discussed above. The beaming fraction is, effectively, the only big unknown in this calculation and it is often useful to quote potentially observable pulsar number estimates (i.e. those obtained before any beaming model has been applied).

The most recent analysis to use the scaling function approach to derive the characteristics of the true normal and millisecond pulsar populations is based on the sample of pulsars within a cylinder of radius $1.5 \mathrm{kpc}$ centred on the Sun (Lyne et al. 1998). The rationale for this cut-off is that, within this region, the selection effects are well understood and easier to quantify 
by comparison with the rest of the Galaxy. These calculations should, at the very least, give reliable estimates for the local pulsar population.

The luminosity distributions obtained from this analysis are shown in Fig. 8. For the normal pulsars, integrating the corrected distribution above $1 \mathrm{mJy} \mathrm{kpc}{ }^{2}$ and dividing by $\pi \cdot(1.5)^{2} \mathrm{kpc}^{2}$ yields a local surface density, assuming Biggs' (1990) beaming model, of $156 \pm 31$ pulsars $\mathrm{kpc}^{-2}$. The same analysis for the millisecond pulsars, assuming a mean beaming fraction of $75 \%$ (Kramer et al. 1998), leads to a local surface density of $38 \pm 16$ pulsars $\mathrm{kpc}^{-2}$ for luminosities above $1 \mathrm{mJy} \mathrm{kpc}^{2}$.
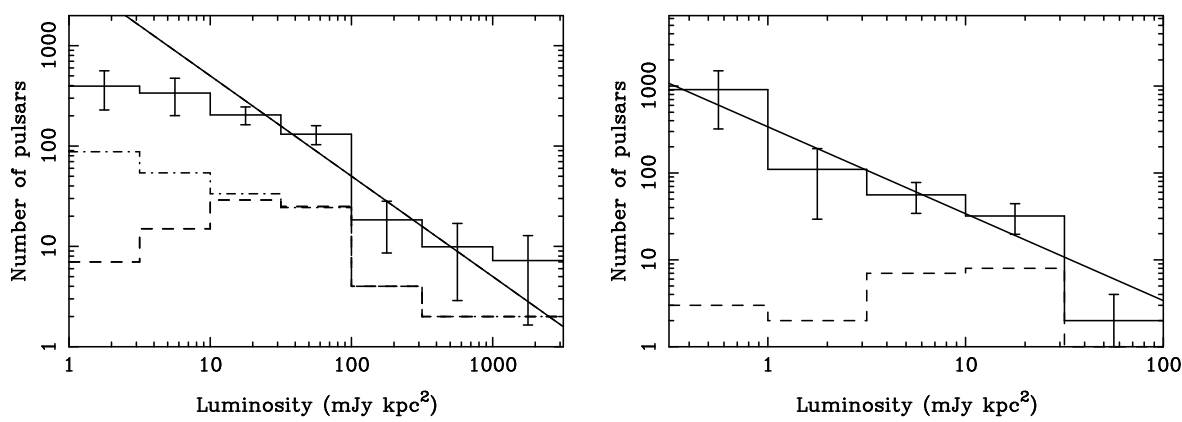

Figure 8. Left: The corrected luminosity distribution (solid histogram with error bars) for normal pulsars. The corrected distribution before the beaming model has been applied is shown by the dot-dashed line. Right: The corresponding distribution for millisecond pulsars. In both cases, the observed distribution is shown by the dashed line and the thick solid line is a power law with a slope of -1 . The difference between the observed and corrected distributions highlights the severe under-sampling of low-luminosity pulsars.

Scaling these local surface densities of pulsars over the whole Galaxy using the factor $1000 \pm 250 \mathrm{kpc}^{2}$ discussed above leads us to an estimate of $(160 \pm 50) \times 10^{3}$ active normal pulsars and $(40 \pm 20) \times 10^{3}$ millisecond pulsars in the Galaxy. These numbers are to be compared with our back-of-theenvelope estimate of 150,000 pulsars (of all types) made at the beginning of this section. The robust analysis using scale factors and more realistic beaming models suggests a larger total population of objects.

\section{Birth Rates}

Simple birth rate estimates based on the above numbers and assuming some mean lifetimes may now be made. For the millisecond pulsars, we recall from the discussion in $\S 2$ that their characteristic ages are a few Gyr. This is corroborated by the observations of white dwarf cooling ages and the effect of asymmetric drift in the proper motions. Since millisecond pulsars cannot, by definition, be older than the age of the Galactic disk itself (which we take to be $10 \mathrm{Gyr}$; see e.g. Jimenez, Flynn \& Kotoneva 
1998 and references therein) we set a lower limit on the millisecond pulsar birth rate of $40,000 / 10^{10}=4 \times 10^{-6} \mathrm{yr}^{-1}$. We discuss this in detail in $\S 7$.

For the normal pulsars, which are clearly much younger than the age of the Galactic disk, we make use of a relatively model-free approach to estimate the birth rate of normal pulsars that was first suggested by Phinney \& Blandford (1981) and applied by Vivekanand \& Narayan (1981). The technique used the scale factor determinations described above to calculate the flow of pulsars from short to long periods - that is the pulsar "current".
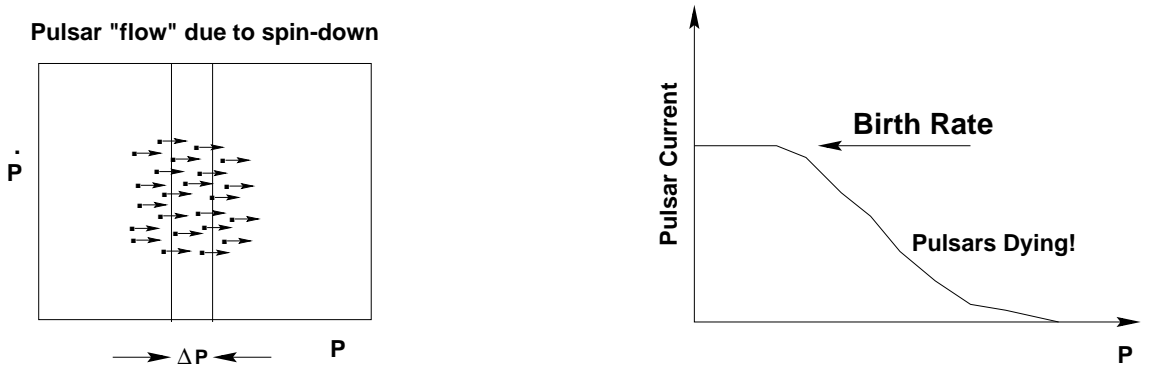

Figure 9. Schematic representation of pulsar current. Left: pulsar flow across the $P-\dot{P}$ plane. Right: pulsar current (number of objects per unit time) as a function of period.

The relevance of pulsar current theory to birth rate calculations is shown in Fig. 9 - a sketch of the expected situation for a pulsar population born with short initial spin periods. Following the initial "plateau", there is a steady decline (reflecting luminosity and/or beaming evolution) until pulsars no longer radiate sufficient energy to be detected. The birth rate of this population is then simply proportional to the height of the plateau. The major advantage of this approach over previous methods is that it assumes nothing about the nature of pulsar spin-down and in this sense is model-free. The only two assumptions underlying the pulsar current theory are: (1) pulsars are spinning down to longer periods; (2) the population has reached steady state i.e. the mean age of the population is much younger than the lifetime of the Galaxy. It should be clear by now that both these assumptions are justified for normal pulsars in the disk of the Galaxy. As a result, the pulsar current analysis does not require any a-priori knowledge of the mean lifetime of the population to estimate the birth rate.

When Vivekanand \& Narayan (1981) first calculated the current, they found a discontinuity in the distribution which showed a significant increase in the current at a period of $\sim 0.5 \mathrm{~s}$. They concluded that, in order to account for this anomaly, a large number of pulsars must be "injected" into the population with periods of about $0.5 \mathrm{~s}$, clearly challenging the conventional view that radio pulsars begin their lives with short periods.

A number of authors have investigated the injection issue further, with contrasting results. Narayan (1987) and Narayan \& Ostriker (1990) un- 
dertook detailed simulations and presented evidence in favour of injection. Lorimer et al. (1993), however, found little evidence to support these claims.

Regardless of whether there is injection into the radio pulsar sample, the discovery of long-period (5-12 s) anomalous X-ray pulsars in supernova remnants (see e.g. Mereghetti's review), which are apparently radio-quiet, has important implications for the spin periods and magnetic fields of neutron stars. It is likely that the anomalous X-ray pulsars will radically change our picture of neutron star birth properties (Gotthelf \& Vasisht 2000)

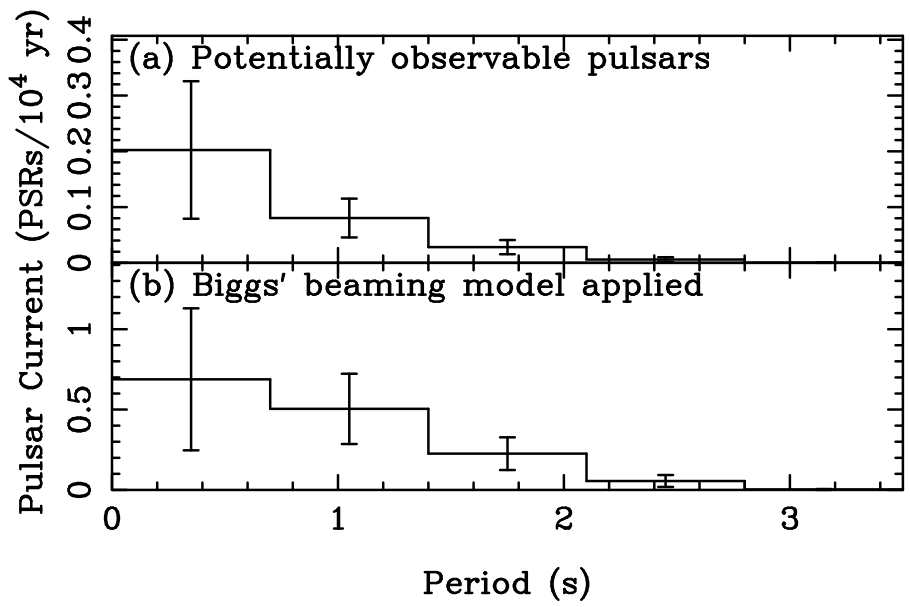

Figure 10. The results of the pulsar current analysis carried out by Lyne et al. (1998). The top panel (a) shows the current derived with no corrections for beaming. The lower panel (b) shows the data after applying Bigg's (1990) beaming model.

The results of the pulsar current analysis by Lyne et al. (1998) are shown in Fig. 10. To derive the birth rate from this figure, we take the pulsar current from the first bin (0.007 pulsars per century assuming Biggs' beaming model) and divide this by $\pi \cdot(1.5)^{2} \mathrm{kpc}^{2}$ to scale it to unit surface area. Multiplying this number by the local-to-Galactic scale factor of $1000 \pm$ $250 \mathrm{kpc}^{2}$ discussed above, the overall birth rate of normal pulsars is $1.0 \pm 0.7$ pulsars per century, about 2500 times larger than the lower limit obtained for the millisecond pulsars. This highly significant difference in the birth rates of the two species is further indication that the vast majority of binary systems disrupt at the moment of the first supernova.

The relatively large error bars in the birth rate estimate for normal pulsars reflects the model-free nature of the pulsar current analysis adopted. Other authors (see e.g. Narayan 1987) have attempted to reduce the uncertainties by making additional assumptions about pulsar luminosity. Whilst this is a commendable approach, there is presently no convincing evidence to support these assumptions. The interested reader is referred to $\S 4.2$ of Lorimer et al. (1993) for further discussion which is not repeated here. 


\section{Summary, Implications and Suggestions for Future Work}

Having made a lengthy tour, with many diversions and stops on the way, we have finally achieved our goal of obtaining (some) birth rate estimates for the neutron star population. In this closing section, we discuss the implications of these results in the context of the proposed progenitor populations.

\subsection{NORMAL PULSARS AND CORE-COLLAPSE SUPERNOVAE}

From the results of Lyne et al. (1998), a normal pulsar is born on average once every 60-330 yr in the Galaxy. More recently, Brazier \& Johnston (1999) have derived a rate of one birth every $90 \mathrm{yr}$ from a local sample of $\mathrm{X}$-ray detected neutron stars. This latter value is independent of the radio pulsar beaming model. Based on an analysis of historical supernovae by Tammann (see $\$ 8.3 .2$ of van den Bergh and Tammann 1991) the rate of Galactic supernovae is one event every 10-30 yr. Thus, in the sense that the radio pulsar production rate does not significantly exceed the supernova rate, this is consistent with our expectation from the standard model that every normal radio pulsar we see began its life in a supernova. Two important caveats are intimately entwined with this statement:

(1) The radio pulsar birth rate applies to objects with luminosities above $1 \mathrm{mJy} \mathrm{kpc}^{2}$, and needs to be boosted by some factor if a significant part of the population is born with lower luminosities. The study of Lyne et al. (1998) shows that the radio luminosity function is flattening between 1 and $10 \mathrm{mJy} \mathrm{kpc}^{2}$ (Fig. 8). Lorimer et al. (1993) suggested that there may be no need to have pulsars born with luminosities below $10 \mathrm{mJy} \mathrm{kpc}^{2}$. The implication of this is that the faint pulsars have undergone a significant luminosity evolution since birth. Further studies of this issue are warranted. (2) The supernova rate is based on a sample which is a mixture of all supernovae types. It should scaled by the fraction of core-collapse supernovae. Based on the death rate of stars more massive than $6.5 \mathrm{M}_{\odot}$, From the discussion in $\S 8.5 .1$ of van den Bergh and Tammann (1991), one can deduce the Galactic rate of core-collapse supernova to be event every 10-50 yr.

Assuming that few radio pulsars are born with exceedingly low luminosities, it seems that the birth rate of radio pulsars is significantly below the rate of core-collapse supernovae. Presently, there seems to be little evidence that the birth rates of soft gamma-ray repeaters (Kouveliotou et al. 1994) and anomalous X-ray pulsars (Gotthelf \& Vasisht 1998) will add significantly to the rates discussed here, although further studies of the selection effects imposed on these neutron star populations are warranted. Future attempts to place tighter constraints on the pulsar birth rate (for example based on a statistical analysis of the Parkes multibeam survey results) should also clarify this situation. 


\subsection{MILLISECOND PULSARS AND LOW-MASS X-RAY BINARIES}

As derived in $\S 6$, based on the analysis of Lyne et al. (1998), and assuming a beaming fraction of $75 \%$, a lower limit to Galactic birth rate of millisecond pulsars is $4 \times 10^{-6} \mathrm{yr}^{-1}$. This number is based on a sample of 18 sources within $1.5 \mathrm{kpc}$ of the Sun with $400 \mathrm{MHz}$ luminosities $\gtrsim 1 \mathrm{mJy} \mathrm{kpc}^{2}$. The entire sample from this paper is summarised in Table 1. This includes the aforementioned objects, plus a further three solitary millisecond pulsars with luminosities below 1 mJy $\mathrm{kpc}^{2}$ that also lie within $1.5 \mathrm{kpc}$.

\begin{tabular}{llllllll}
\hline PSR & Type & $\begin{array}{l}L_{400} \\
\mathrm{mJy} \mathrm{kpc}^{2}\end{array}$ & $\begin{array}{l}\text { Scale } \\
\text { Fact }\end{array}$ & PSR & Type & $\begin{array}{l}L_{400} \\
\mathrm{mJy} \mathrm{kpc}^{2}\end{array}$ & $\begin{array}{l}\text { Scale } \\
\text { Fact }\end{array}$ \\
\hline $\mathrm{J} 0034-0534$ & $\mathrm{sB}$ & 16 & 6 & $\mathrm{~J} 1744-1134$ & $\mathrm{Si}$ & 0.4 & 210 \\
$\mathrm{~J} 0437-4715$ & $\mathrm{sB}$ & 19 & 3 & $\mathrm{~J} 1804-2717$ & $\mathrm{sB}$ & 14 & 2 \\
$\mathrm{~J} 0711-6830$ & $\mathrm{Si}$ & 11 & 7 & $\mathrm{~B} 1855+09$ & $\mathrm{sB}$ & 12 & 3 \\
$\mathrm{~J} 1012+5307$ & $\mathrm{sB}$ & 5 & 9 & $\mathrm{~J} 2019+2425$ & $\mathrm{lB}$ & 2 & 40 \\
$\mathrm{~J} 1022+1001$ & $\mathrm{sB}$ & 5 & 10 & $\mathrm{~J} 2033+1734$ & $\mathrm{lB}$ & 10 & 6 \\
& & & & & & & \\
$\mathrm{~J} 1024-0719$ & $\mathrm{Si}$ & 0.6 & 180 & $\mathrm{~J} 2051-0827$ & $\mathrm{sB}$ & 9 & 7 \\
$\mathrm{~B} 1257+12$ & $\mathrm{Pl}$ & 8 & 5 & $\mathrm{~J} 2124-3358$ & $\mathrm{Si}$ & 0.4 & 520 \\
$\mathrm{~J} 1455-3330$ & $\mathrm{lB}$ & 5 & 9 & $\mathrm{~J} 2145-0750$ & $\mathrm{sB}$ & 18 & 2 \\
$\mathrm{~J} 1713+0747$ & $\mathrm{lB}$ & 15 & 4 & $\mathrm{~J} 2229+2643$ & $\mathrm{lB}$ & 13 & 5 \\
$\mathrm{~J} 1730-2304$ & $\mathrm{Si}$ & 4 & 10 & $\mathrm{~J} 2317+1439$ & $\mathrm{sB}$ & 68 & 2 \\
& & & & $\mathrm{~J} 2322+2057$ & $\mathrm{Si}$ & 1 & 70 \\
\hline
\end{tabular}

TABLE 1. The sample of 21 millisecond pulsars used by Lyne et al. (1998). For each pulsar, we list the type of system, $400 \mathrm{MHz}$ luminosity and derived scale factor. Type is either: sB (short-period binary), lB (long-period binary), Si (single pulsar) or Pl (planetary system). The boundary between orbital periods of $\mathrm{sB}$ and $\mathrm{lB}$ systems is 25 days. Note that this sample is confined to a cylinder of radius $1.5 \mathrm{kpc}$ centred on the Sun and does therefore not contain the few more distant millisecond pulsars (e.g. PSR B1937+21).

In the context of the standard formation scenario discussed in $\S 2$, the binary millisecond pulsars descend from low-mass X-ray binaries. The sum of the scale factors of just the binary systems (sB and lB types) listed in Table 1, is 108. From the sample definition of Lyne et al., this translates to a local surface density of $108 /\left(\pi \cdot(1.5)^{2}\right) \simeq 15$ binary millisecond pulsars $\mathrm{kpc}^{-2}$. Assuming, as before, that these pulsars beam to $75 \%$ of the sky, and using the Galactic scale factor of $1000 \mathrm{kpc}^{2}$ (Ratnatunga \& van den Bergh 1989; see $§ 5$ ), we estimate the Galactic population of binary millisecond pulsars to be 20,000. Taking the maximum lifetime of this population to be 10 Gyr results in a lower limit to the birth rate for this population of 
$2 \times 10^{-6} \mathrm{yr}^{-1}$. This number is comfortably below the birth rate for low-mass X-ray binaries $\left(\gtrsim 7 \times 10^{-6} \mathrm{yr}^{-1}\right)$ derived by Coté \& Pylyser (1989).

In the original millisecond pulsar birth rate analysis, Kulkarni \& Narayan (1988) found a significant discrepancy between the birth rate of shortorbital period ( $\lesssim 25$ day) millisecond pulsar binaries and their progenitor low-mass X-ray binary systems, i.e. the pulsar birth rate was two orders of magnitude too large. Based on the larger sample here, we can easily re-investigate this issue. Repeating the above analysis for the 9 shortperiod (sB) binaries in Table 1, we find the local surface density to be $44 /\left(\pi \cdot(1.5)^{2}\right) \simeq 6$ short-period binaries $\mathrm{kpc}^{2}$. Making the same beaming and Galactic scaling correction as in the previous calculation, we find a Galactic birth rate for these sources of $\gtrsim 8 \times 10^{-7} \mathrm{yr}^{-1}$. Whilst this is nominally a factor of at least four larger than Coté \& Pylyser's estimate for short-period low-mass X-ray binary systems $\left(2 \times 10^{-7} \mathrm{yr}^{-1}\right)$, the discrepancy does not seem to be as pronounced as previously thought. Based on the uncertainties in both birth rates (factors of a few), one could conclude that the populations are consistent with each other.

Although all seems to be well with the connection between low-mass X-ray binaries and millisecond pulsars, we should re-iterate the statement made in $\S 2$ that it is presently still something of a mystery how the isolated millisecond pulsars are formed. Based on the 6 single millisecond pulsars (Si) in Table 1, we deduce a Galactic birth rate of $2 \times 10^{-5} \mathrm{yr}^{-1}$. This is significantly larger than the birth rates for the binary pulsars and is primarily a reflection of the low luminosities observed for PSRs J1024-0719, J17441134 and J2124-3358 which results in relatively large scale factor estimates. Indeed, these pulsars dominate the lowest bin in the luminosity function (Fig. 8). It is presently marginally significant that solitary millisecond pulsars have, on average, lower luminosities than binary millisecond pulsars (Bailes et al. 1997; Kramer et al. 1998). If confirmed by future discoveries, this may be a clue to the origin of these mysterious objects.

As a final remark, we estimate from Table 1 that the Galactic population of millisecond pulsars with planetary systems is of order 900. This estimate is based upon only one object and therefore has a $100 \%$ uncertainty! It does appear, from the lack of discoveries of similar systems, that the birth rate of pulsar planetary systems in the Galactic disk is rather small.

\section{Acknowledgements}

I wish to thank the organisers of this meeting for putting together an exciting programme, and providing a splendid venue in which to hold it. Many thanks to Mike Davis and Fernando Camilo for extremely useful comments on an earlier version of this manuscript. 


\section{References}

Alpar M. A., Cheng A. F., Ruderman M. A., Shaham J., 1982, Nat, 300, 728

Backer D. C., 1970, Nat, 228, 42

Bailes M., 1989, ApJ, 342, 917

Bailes M. et al., 1997, ApJ, 481, 386

Bhattacharya D., Wijers R. A. M. J., Hartman J. W., Verbunt F., 1992, A\&A, 254, 198

Biggs J. D., 1990, MNRAS, 245, 514

Bisnovatyi-Kogan G. S., Komberg B. V., 1974, SA, 18, 217

Blaauw A., 1961, Bull. Astr. Inst. Netherlands, 15, 265

Brazier K. T. S., Johnston S., 1999, MNRAS, 305, 671

Camilo F. et al. 2000, Pulsar Astronomy - 2000 and Beyond, (astro-ph/9911185)

Chakrabarty D., Morgan E. H., 1998, Nat, 394, 346

Coté J., Pylyser E. P. H., 1989, A\&A, 218, 131

Dewey R. J. et al. 1984, in Reynolds S., Stinebring D., eds, Millisecond Pulsars. p. 234

Emmering R. T., Chevalier R. A., 1989, ApJ, 345, 931

Flannery B. P., van den Heuvel E. P. J., 1975, A\&A, 39, 61

Gotthelf E. V., Vasisht G., 1998, New Astronomy, 3, 293

Gotthelf E. V., Vasisht G., Pulsar Astronomy - 2000 and Beyond, (astro-ph/9911344)

Hansen B., Phinney E. S., 1997, MNRAS, 291, 569

Hills J. G., 1983, ApJ, 267, 322

Hulse R. A., Taylor J. H., 1975, ApJ, 195, L51

Jimenez R., Flynn C., Kotoneva E., 1998, MNRAS, 299, 515

Kouveliotou C. et al. 1994, Nat, 368, 125

Kramer M. et al. 1998, ApJ, 501, 270

Kulkarni S. R., Narayan R., 1988, ApJ, 335, 755

Kundt W., 1992, in Hankins T. H., Rankin J. M., Gil J., eds, IAU Colloquium 128. Pedagogical University Press, Zielona Góra, Poland, p. 86

Lawson K. D., Mayer C. J., Osborne J. L., Parkinson M. L., 1987, MNRAS, 225, 307

Lorimer D. R., Bailes M., Harrison P. A., 1997, MNRAS, 289, 592

Lorimer D. R., Bailes M., Dewey R. J., Harrison P. A., 1993, MNRAS, 263, 403

Lorimer D. R., Yates J. A., Lyne A. G., Gould D. M., 1995, MNRAS, 273, 411

Lyne A. G., Manchester R. N., 1988, MNRAS, 234, 477

Lyne A. G. et al., 1998, MNRAS, 295, 743

Narayan R., Ostriker J. P., 1990, ApJ, 352, 222

Narayan R., Vivekanand M., 1983, A\&A, 122, 45

Narayan R., 1987, ApJ, 319, 162

Nice D. J., 1999, ApJ, , 927

Phinney E. S., Blandford R. D., 1981, MNRAS, 194, 137

Ratnatunga K. U., van den Bergh S., 1989, ApJ, 343, 713

Ritchings R. T., 1976, MNRAS, 176, 249

Scheuer P. A. G., 1968, Nat, 218, 920

Shapiro S. L., Teukolsky S. A., 1983, The physics of Compact Objects. Wiley, New York

Smarr L. L., Blandford R., 1976, ApJ, 207, 574

Spruit H., Phinney E. S., 1998, Nat, 393, 139

Stollman G. M., 1987, A\&A, 178, 143

Tauris T. M., Manchester R. N., 1998, MNRAS, 298, 625

Toscano M., Sandhu J. S., Bailes M., Manchester R. N., Britton M. C., Kulkarni S. R., Anderson S. B., Stappers B. W., 1999, MNRAS, 307, 925

van den Bergh S., Tammann G. A., 1991, ARAA, 29, 363

van Kerkwijk M. H., 1996, in Johnston S., Walker M. A., Bailes M., eds, Pulsars: Problems and Progress, IAU Coll 160. Astronomical Society of the Pacific, San Francisco, p. 489

Vivekanand M., Narayan R., 1981, JAPAS, 2, 315

Wijnands R., van der Klis M., 1998, Nat, 394, 344

Young M. D., Manchester R. N., Johnston S., 1999, Nat, 400, 848 